\title{
Examinations of identity invariance in facial expression adaptation
}

\author{
Melissa Ellamil, Joshua M. Susskind, And Adam K. Anderson \\ University of Toronto, Toronto, Ontario, Canada
}

\begin{abstract}
Faces provide a wealth of information essential to social interaction, including both static features, such as identity, and dynamic features, such as emotional state. Classic models of face perception propose separate neural-processing routes for identity and facial expression (Bruce \& Young, 1986), but more recent models suggest that these routes are not independent of each other (Calder \& Young, 2005). Using a perceptual adaptation paradigm in the present study, we attempted to further examine the nature of the relation between the neural representations of identity and emotional expression. In Experiment 1, adaptation to the basic emotions of anger, surprise, disgust, and fear resulted in significantly biased perception away from the adapting expression. A significantly decreased aftereffect was observed when the adapting and the test faces differed in identity. With a statistical model that separated surface texture and reflectance from underlying expression geometry, Experiment 2 showed a similar decrease in adaptation when the face stimuli had identical underlying prototypical geometry but differed in the static surface features supporting identity. These results provide evidence that expression adaptation depends on perceptual features important for identity processing and thus suggest at least partly overlapping neural processing of identity and facial expression.
\end{abstract}

Humans are social beings, and face perception is an essential aspect of social cognition. Faces provide a great amount of information critical to social interaction, including both static features, such as information about a person's age, gender, race, and identity, and dynamic or changeable features, such as those that communicate one's internal emotional state (Calder \& Young, 2005; Haxby, Hoffman, \& Gobbini, 2000). Classic models of face perception (Bruce \& Young, 1986) propose separate, specialized, and parallel processing routes for these distinct feature sets - in particular, the recognition of identity and facial expression. A functional analysis suggests that it is advantageous to be able to identify a person regardless of their facial expression, with an abstract, invariant representation of an identity being transferred across different facial expressions much as it transfers across different viewpoints. Conversely, since it is also useful to be able to infer the same emotional states from similar expressions of different individuals, abstract, invariant representations of different facial expressions need to be transferred across different people.

Supporting this functional segregation, research has shown that distinct neural processes may support identity and facial expression analyses. A double dissociation between the ability to identify faces and the ability to interpret expressions has been observed in both brain-damaged patients (e.g., Bruyer et al., 1983; Kurucz \& Feldmar, 1979; Kurucz, Feldmar, \& Werner, 1979) and healthy subjects (Bruce, 1986). The separation between the processing of static invariant (e.g., identity) and dynamic variable (e.g., expression) aspects of faces has also been observed in neurophysiological studies, with preferential processing of the former by the inferior temporal gyrus (the middle fusiform gyrus in humans; Grill-Spector, Knouf, \& Kanwisher, 2004; Kanwisher, McDermott, \& Chun, 1997) and preferential processing of the latter by the superior temporal sulcus (STS; Haxby et al., 2000; Narumoto, Okada, Sadato, Fukui, \& Yonekura, 2001; Winston, Henson, FineGoulden, \& Dolan, 2004). In addition, neuropsychological and neuroimaging evidence suggests that the recognition of categorically distinct prototypical emotions depends on further differentiated neural representations, such as the amygdala for fear (e.g., Adolphs et al., 1999; Anderson, Christoff, Panitz, De Rosa, \& Gabrieli, 2003; Calder, Lawrence, \& Young, 2001) and the right anterior insula for disgust (e.g., Anderson et al., 2003; Phillips et al., 1998).

Recent behavioral studies, however, have suggested that the neural representations and processes of identity and facial expression may not be entirely independent of each other. Variations in identity interfere with the speed of expression classification (Ganel \& Goshen-Gottstein, 2004; Schweinberger \& Soukup, 1998). Viewing different expressions improves identity recognition of familiar faces (Kaufmann \& Schweinberger, 2004) and learning the identity of unfamiliar faces (Baudouin, Gilibert, Sansone, \& Tiberghien, 2000). Computational analyses of identity and facial expression have further shown the potential overlap between identity and expression representations. Employing principal component analysis, Calder, Burton, Miller, Young, and Akamatsu (2001) found substantial overlap between the components important for identity and expression 
discrimination in a statistical model, so that identity discrimination was still possible when analysis was restricted to components selective for expression discrimination and vice versa. Neuroimaging studies have also shown a large overlap in activation patterns and associated processes drawn upon during identity and facial expression recognition tasks (e.g., LaBar, Crupain, Voyvodic, \& McCarthy, 2003). Facial speech processing, another changeable aspect of the face, has similarly been shown to interact with identity processing (Walker, Bruce, \& O'Malley, 1995).

Adaptation paradigms provide a way of further investigating the neural representations of faces (Fox \& Barton, 2007b) and refining current theories on the independence of identity and facial expression from one another or their dependence on each other. One interpretation of adaptation is the decrease over time in the responsiveness of a neural representation to a constant stimulus, which is believed to fatigue the neural mechanisms associated with the processing of a particular feature of that stimulus (Grill-Spector, Henson, \& Martin, 2006; Köhler \& Wallach, 1944). The result is a biased perception of the stimulus toward its opposite (e.g., figural aftereffects; Webster, Kaping, Mizokami, \& Duhamel, 2004). Adaptation has been observed for a variety of visual features, such as size, orientation, curvature, spatial frequency, motion, and complex and natural images (Webster \& MacLin, 1999). In particular, aftereffects have now also been observed with distorted faces (e.g., contracted or expanded, changed spatial frequency) that are size and retinal position invariant (e.g., Leopold, O’Toole, Vetter, \& Blanz, 2001; Rhodes, Jeffery, Watson, Clifford, \& Nakayama, 2003; Zhao \& Chubb, 2001). Higher order adaptation to natural facial categories, such as gender, ethnicity, attractiveness, and identity, has also been shown (e.g., Leopold et al., 2001; Rhodes et al., 2003; Webster et al., 2004).

In addition to static facial features, adaptation has been shown for dynamic features, such as eye gaze (Calder et al., 2007; Jenkins, Beaver, \& Calder, 2006) and facial expression (Fox \& Barton, 2007b; Hsu \& Young, 2004; Webster et al., 2004). Specifically, repeated exposure to an expression significantly biased perception away from the adapting expression. For example, repeated exposure to a face expressing happiness will reduce sensitivity to perceiving happiness in facial expression mixtures (e.g., a happinessdisgust morphed expression). Thus, studies have separately shown that adaptation can be used to interrogate the neural representations supporting both identity and expression recognition. However, little work has focused on the interaction of identity and expression in facial adaptation, which would have important consequences for examining the independence of neural representations of identity and expression.

Recently, Fox and Barton (2007b) investigated whether facial expression adaptation generalizes across different identities. If facial expression adaptation results from modulation of identity-independent/expression-dependent representations, they should be resilient to changes in identity between the adapting and the test stimuli. Expression aftereffects, although present, were significantly diminished when the adapting and the test stimuli had differing identities. These results are consistent with some com- mon neural process supporting identity and expression processing. However, it is unclear to what extent reduced expression adaptation is due to changes in identity or expression features. Any decreases in identity-expression cross-adaptation not only may reflect differences in the static features supporting identity, but also may originate in differences in the dynamic features supporting expression appearance. For instance, individuals may differ in the degree to which their expressions match underlying prototype geometry. Differences in expression geometry across individuals may preclude undiminished adaptation.

To examine whether expression aftereffects are identity invariant - that is, are preserved when the identity is altered between presentations of the adapting and the test stimuliwe attempted to more precisely determine the cause of the reduced expression aftereffect by carefully controlling facial features associated with identity and expression with a visual statistical model of facial appearance (Craw \& Cameron, 1991). In Experiment 1, we examined identityexpression cross-adaptation, using emotions thought to depend on specialized limbic and paralimbic neural substrates. Experiment 2 analyzed whether differences in expression geometry or features supporting identity accounted for reduced expression adaptation across identities. This was achieved by generating novel realistic facial stimuli via the factoring of face images into their geometrical (i.e., shape) and surface (i.e., texture and reflectance) properties (Craw \& Cameron, 1991). This afforded a novel computational method for mapping different facial identities onto identical prototypical expression geometry and a direct test of whether expression adaptation is robust to changes in surface identity when expression form is held constant.

\section{EXPERIMENT 1}

\section{Method}

Participants. Thirty-two participants contributed data for analysis (20 of them female; age, $M=19.56$ years, $S D=2.20$ years). All were undergraduate students at the University of Toronto who volunteered for experimental credit toward their introduction to psychology course or were recruited via posters and were paid $\$ 10$ for their participation. All had normal or corrected-to-normal vision. All were unaware of the purpose of the experiment, and informed consent was obtained from each participant before the experiment started, in accordance with the ethics guidelines at the university.

Stimuli and Apparatus. Grayscale images of two female individuals were obtained from Ekman and Friesen's (1976) Pictures of Facial Affect (POFA) data set. Each individual posed for four different expressions: anger, disgust, fear, and surprise. The two individuals were selected because they yielded the highest facial expression recognition rates in the POFA data set. The four emotions were chosen on the basis of behavioral findings that the members of pairs consisting of anger and surprise and of disgust and fear are judged as most dissimilar to one another (Susskind, Littlewort, Bartlett, Movellan, \& Anderson, 2007). The images were processed for experimental display using MATLAB 7.0. All the faces were cut out from the background image, using a polygonal region defined by a consistent facial contour labeling scheme. Pixels inside the polygonal region defining the face were histogram equalized to remove variability due to lighting differences across faces. All of the faces were then commonly aligned to remove global differences in size, rotation, and translation, using a generalized Procrustes transformation (Goodall, 1991) on a set of 68 feature point locations including the contour, eyebrows, eyes, nose, and mouth. Each face was 
cropped to a common face box and was resized to $337 \times 328$ pixels, maintaining the aspect ratio of the common face box.

Test faces for the two-alternative forced choice task before and after the adaptation phase were drawn from morph continua consisting of 100 images each, with the first image (left end, $0 \%$ ) of a facial expression gradually turning into another expression by the 100th image (right end, 100\%). The category boundary or neutral point was the percentage or morph level at which the image would be equally likely to be perceived as either the left or the right end of the continuum. The anger and surprise images of the same-identity face were morphed together using FantaMorph 3.0, and the morphing sequence was stored as 100 frames of a movie file. The disgust and fear images of the same-identity face were morphed in the same way.

The experimental program was written using Visual Basic 6.0. A PC was used to present the stimuli on a uniform gray background, with the monitor mounted approximately $50 \mathrm{~cm}$ in front of the participants. The participants pressed either a red or a green button on the keyboard to make a two-alternative forced choice response but were not given specific instructions for which hand to use. All the instructions appeared on the monitor.

The independent variables were the continuum from which the test faces were drawn (anger-surprise or disgust-fear; between subjects), the identity of the adapting face (same as or different from the continuum; between subjects), and the adapting expression (anger/disgust, left side of continuum; surprise/fear, right side of continuum; within subjects), resulting in four groups (continuum/ adapting-identity): anger-surprise/same-identity, anger-surprise/ different-identity, disgust-fear/same-identity, and disgust-fear/ different-identity. The dependent variable was the difference between the pre- and the postadaptation category boundaries (i.e., post minus pre). A negative value meant that the boundary shifted toward the left end of the continuum (anger or disgust), whereas a positive value meant that it shifted toward the right end (surprise or fear).

Procedure. The participants carried out the experiment individually and were randomly assigned to the four groups. The experimental procedure is illustrated in Figure 1. During the preadaptation phase, a test stimulus (i.e., an individual frame from a continuum) was presented for $500 \mathrm{msec}$ on each trial, followed by a prompt screen with two response options, which was shown until a response was made. The participant classified the image as expressing one of two emotions, which corresponded to the two ends of the continuum, by pressing either the red (left choice) or the green (right choice) button. A double, randomly interleaved staircase procedure (Cornsweet, 1962) was used to present the stimuli and to estimate the continuum category boundary (i.e., the morph level at which either of the two responses was equally likely; Webster et al., 2004). Each of the two staircases started at one end of the continuum or the other. The morph level of the test stimulus on each trial was increased or decreased in steps of two, depending on the response to the previous trial, for each of the two staircases. If, for example, the image was selected from the left end of the anger-surprise continuum and the participant judged anger, the next image would be selected from two steps up (i.e., toward the right, or surprise, end). The image would keep increasing in morph level (i.e., moving to the right) until the participant judged surprise, at which point the staircase would reverse (counted as one reversal) and the next image would be two steps down (i.e., back toward the left, or anger, end). The same dependency would occur for the staircase starting from the right/surprise end. The staircase procedure terminated after 12 reversals for each staircase. The morph levels at which the 12 reversals occurred within each of the two staircases were averaged, and the two resulting values were averaged to determine a single value, which was taken as the category boundary (neutral point) between the two expressions.

During the adaptation phase, the participant was instructed to look at one of the original images from one end of the continuum continuously for $180 \mathrm{sec}$ but was not given specific fixation instructions. During the postadaptation phase, the participant again classified test stimuli into one of two emotions to reestimate the reversal points, using the procedure from the preadaptation phase, but with the adapting image shown for $5 \mathrm{sec}$ between each of the test images. There was an interstimulus interval of $250 \mathrm{msec}$ between the adapting and the test stimuli, during which a uniform gray background was shown. After a short break, the three phases were repeated with the adapting face chosen from the other end of the continuum. The order in which the two adaptation blocks were presented was randomized across participants.

\section{Results}

An alpha level of .05 was used for all statistical tests. A three-way ANOVA for mixed designs, with continuum, adapting identity, and adapting expression as factors, tested for the effects of identity and facial expression on adaptation. The dependent measure was the difference between the pre- and the postadaptation category boundaries (post minus pre). The effect of identity on expression adaptation would be revealed through an identity $\times$ expression interaction. Consistent with expression adaptation, there was a robust main effect of adapting expression $[F(1,28)=326.56$, $p<.001]$, with adaptation to the left end of the continuum shifting the category boundary to the left $(M=-16.63$, $S D=10.57)$ and adaptation to the right end $(100 \%)$ shifting it to the right $(M=18.39, S D=9.15)$. For instance, adapting to anger or disgust resulted in seeing less anger or disgust in morphed faces. Thus, the adapting expression shifted the categorical boundary closer to the matching expression prototype. There was a significant interaction of identity and expression $[F(1,28)=17.59, p<.001]$, with adaptation to a different identity leading to significantly smaller boundary shifts (i.e., expression aftereffects) than did adaptation to the same identity (Figure 2). Restricting analysis to the same-identity condition revealed highly robust adaptation $[F(1,14)=302.09, p<.001]$. Although significantly diminished, there remained evidence of sig-
Preadaptation

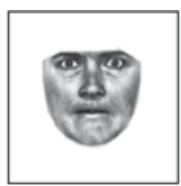

Test stimulus $500 \mathrm{msec}$

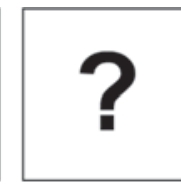

Two-alternative forced choice decision
Adaptation

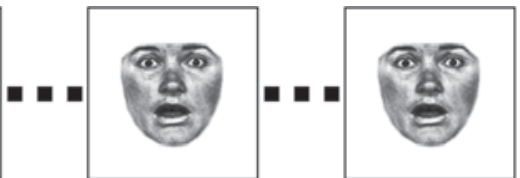

Adapting stimulus

$180 \mathrm{sec}$
Adapting stimulus $5 \mathrm{sec}$
Postadaptation

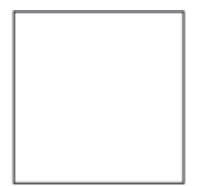

ISI

$250 \mathrm{msec}$

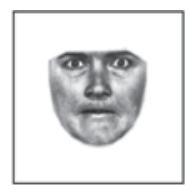

Test stimulus $500 \mathrm{msec}$

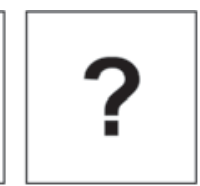

Two-alternative forced choice decision

Figure 1. Schematic of Experiment 1 procedure. ISI, interstimulus interval. 
nificant expression aftereffects for the different-identity trials as well $[F(1,14)=81.63, p<.001]$.

\section{Discussion}

Consistent with previous facial expression adaptation studies (Fox \& Barton, 2007b; Hsu \& Young, 2004; Webster et al., 2004), the perception of facial expressions was reliably and noticeably biased by prolonged exposure to images of facial expressions. Fatiguing the representation of a facial expression led to reduced sensitivity to that expression, decreasing the likelihood that the expression would be perceived and increasing the likelihood that a competing expression would be perceived. Although this expression aftereffect occurred both when the identities of the adapting and test faces were the same and when they were different, a much more pronounced aftereffect was observed when the adapting and the test faces were from the same individual. This is consistent with the reduced expression adaptation across identities reported by Fox and Barton (2007b), as well as with partial sparing of the adaptation effect, suggesting a significant degree of overlap and/or interaction in the processing of neural representations of identity and expression.
One potential complication with this interpretation is that identical adaptation and test images were used in the same-identity condition, following prior work on expression adaptation (Hsu \& Young, 2004; Webster et al., 2004). Thus, differences across identities may reflect differences in low-level visual features, rather than high-level featural similarity. However, it is unlikely that adaptation reflects redundancy in local luminance, since the participants were given ample time to move their eyes during adaptation. Nevertheless, we further explored this issue in Experiment 2.

Another plausible explanation for reduced expression adaptation across individuals is that individuals differ in the extent to which they convey emotion when producing an expression. Different identity exemplars may present substantial variance in the intensity and precise form of expression features and, thus, may match expression category prototypes to differing degrees. As such, it may be differences in the changeable features that support expression geometry in different individuals that accounts for decreased expression adaptation across identities, and not identity change itself.

To explore the contributions of the above, we developed faces for which expression shape (i.e., feature geometry)

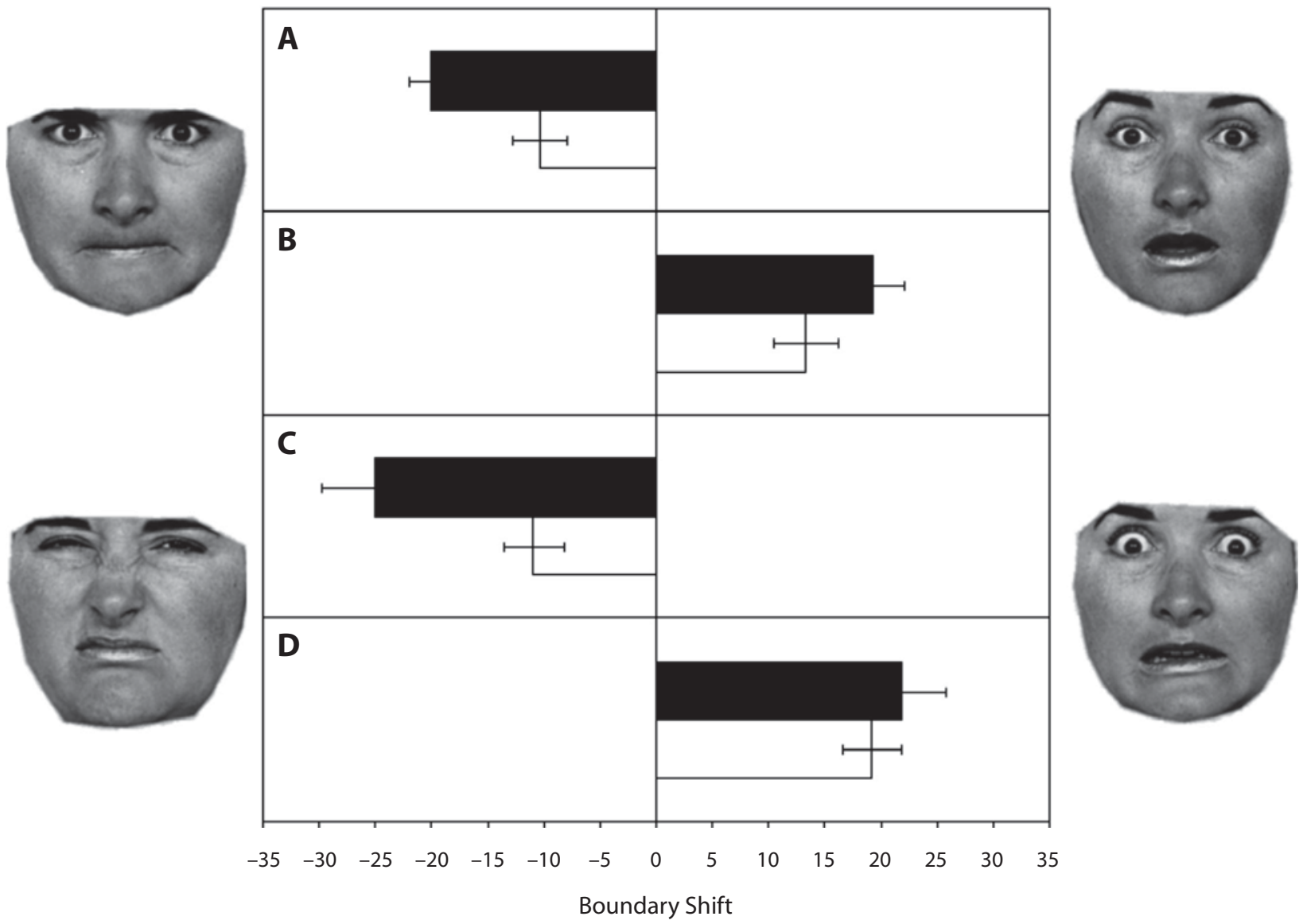

Figure 2. Mean difference between the pre- and the postadaptation category boundaries in Experiment 1 for the anger-surprise (A, B) and disgust-fear $(C, D)$ continua as a function of adapting expression $(A=$ anger, $B=$ surprise, $C=$ disgust, $D=$ fear $)$ and identity (black bars, same as the test face; white bars, different from the test face). A negative value represents a boundary shift toward the left end of the continuum (anger or disgust), whereas a positive value represents a shift toward the right end (surprise or fear). Bar length represents magnitude of adaptation. Error bars represent the standard errors of the means. 


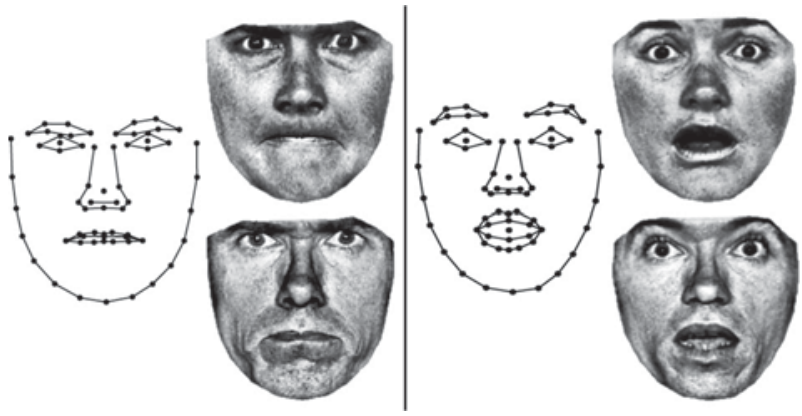

Figure 3. Examples of the anger (left panel) and surprise (right panel) facial warp stimuli used in Experiment 2, with geometrical differences in expression between identities removed but variance in surface reflectance characteristic of each identity retained. The shape schematics in the left and right panels depict the underlying shapes for anger and surprise to which face features were warped.

and surface texture and reflectance could be manipulated separately. If the decrease in expression adaptation across identities was due to individual differences in expression geometry and to corresponding individual differences in neural representation of expression, holding the expression geometry constant should result in expression aftereffects that were the same size regardless of identity. In Experiment 2, we generated images of identical geometry/ shape and warped surface textures from different identity face images onto these shapes (Figure 3). This allowed an examination of whether the identity-related decrease in adaptation reflected changes primarily in identity, while holding expression geometry constant. Expression geometry was computed by averaging the geometries of exemplars in an expression category to more closely align faces with an underlying expression prototype, serving also to limit variance in adaptation across identities. We further examined the influence of a small horizontal distortion of the underlying facial geometry of one of the adapting faces. This allowed a determination of whether greater adaptation for the same identity merely reflected presenting the identical image during the adaptation and test trials. We also examined whether change in gender features, in addition to identity, altered expression adaptation. Finally, in Experiment 2, identity was manipulated as a withinsubjects measure in order to allow greater sensitivity in detecting the effect of identity on expression adaptation.

\section{EXPERIMENT 2}

\section{Method}

Participants. Thirty-two participants contributed data for analysis (22 of them female; age, $M=19.31$ years, $S D=1.38$ years). All were undergraduate students at the University of Toronto who volunteered for experimental credit toward their introduction to psychology course or were recruited via posters and were paid $\$ 10$ for their participation. All had normal or corrected-to-normal vision. All were unaware of the purpose of the experiment, and informed consent was obtained from each participant before the experiment started, in accordance with the ethics guidelines at the university.

Stimuli and Apparatus. Grayscale images of two female and two male individuals $(240 \times 240$ pixels $)$ were obtained from POFA (Ekman \& Friesen, 1976). Although there was no significant difference between the two continua in the first experiment, the anger-surprise continuum displayed numerically largest effects of identity on expression adaptation (see Figure 2) and, thus, was used in Experiment 2. The same image-processing steps as those used to prepare the stimuli for Experiment 1 were performed on the faces for this experiment, with additional steps to remove differences in the facial geometry between faces posing a given expression. The first additional step was to create anger and surprise prototype geometries by averaging each of the 68 feature point locations across all of the faces in the anger and surprise categories, respectively. Then each of the four anger faces and each of the four surprise faces were separately warped to the corresponding prototype anger or surprise shape, using a piecewise affine warp defined on a Delaunay triangulation (Goshtasby, 1986) of the feature points. Warping of expression exemplars was done, rather than warping neutral faces to expression prototype geometry, since the latter required a great deal of distortion of the neutral face, resulting in face stimuli that are highly artificial in appearance. This manipulation resulted in removing geometrical differences between identities posing a particular expression while retaining variance in surface reflectance characteristic of each identity (Craw \& Cameron, 1991). Thus, realistic synthetic faces were created that differed in identity and gender but, overall, held expression geometry constant (Figure 3), rendering a novel stimulus set of matching prototypic expressions of differing identities. In addition, to examine whether greater adaptation for the same identity reflects use of the identical image, we performed a $15 \%$ horizontal compression of the underlying facial geometry of the surprise adapting image (from a width of 240 pixels to one of 204 pixels). A horizontal distortion also resulted in a size change but allowed for a more subtle alteration of the adapting image than did using a complete size change. This prevented the change from being too obvious to the participants, which could have resulted in an explicit or conscious change in their judgments.

The experimental program was written using Visual Basic 6.0. A $\mathrm{PC}$ was used to present the stimuli on a uniform gray background, with the monitor mounted approximately $50 \mathrm{~cm}$ in front of the participants. The participants pressed either a red or a green button on the keyboard to make a two-alternative forced choice response but were not given specific instructions for which hand to use. All the instructions appeared on the monitor.

The independent variables were the adapting expression (anger or surprise; between subjects), the gender of the adapting face (same as or different from the continuum; between subjects), and the identity of the adapting face (same as or different from the continuum; within subjects). The dependent measure was the difference between the pre- and the postadaptation category boundaries (post minus pre).

Procedure. To examine whether facial identity information was retained when warped onto a prototypical expression geometry, prior to the adaptation task, the participants performed an identification task in which they identified each of the adapting faces showing anger or surprise by choosing one out of the four surrounding unprocessed POFA neutral expression images. The same adaptation procedure as that in Experiment 1 was used for this experiment, with a few minor modifications. The double, randomly interleaved staircase procedure still terminated after 12 reversals, but the first 3 reversals led to increments/decrements of eight steps, the next 4 reversals to four steps, and the last 5 reversals to two steps. Before continuing with the second adaptation block, the participants viewed a random sequence of images (each shown for $125 \mathrm{msec}$ over $1 \mathrm{~min}$, to speed up the decay of the adaptation effect) drawn from the set of test images, which were pixelated (cell size $=30 \times 30$ pixels) and randomly flipped horizontally, vertically, or both. The order in which the two adaptation blocks were presented was randomized across participants.

\section{Results}

To see whether warping the face points to the prototype position would retain the identity of the face, a onesample $\chi^{2}$ test analyzed the participants' accuracy in the identification task. Average accuracy $(56.25 \%)$ was sig- 
nificantly greater than chance, or $25 \%\left[\chi^{2}(1, N=64)=\right.$ $33.33, p<.001]$, suggesting that discriminative identity information was conveyed in the surface reflectance properties of the face after identity-specific shape information was removed. This is further illustrated in Figure 3, which shows that altering surface texture and reflectance while holding expression geometry constant yielded faces of distinct identities.

A three-way ANOVA for mixed designs was employed, with adapting expression, adapting identity, and adapting identity's gender as factors. The effect of identity on expression adaptation would be revealed through an identity $\times$ expression interaction. There was a significant main effect of adapting expression $[F(1,28)=9.89, p=.004]$, with adaptation to anger shifting the boundary toward the left, or anger, side $(M=-6.17, S D=9.74)$ and adaptation to surprise shifting the boundary toward the right, or surprise, side $(M=4.44, S D=13.80)$ of the morph continuum. That is, the adapting expression shifted the categorical boundary closer to the expression prototype. Replicating Experiment 1, there was a significant identity $\times$ expression interaction $[F(1,28)=7.61, p=.01]$, with adaptation to an identity different from that of the test faces leading to smaller boundary shifts (i.e., expression aftereffects) than did adaptation to the same identity (Figure 4). There was no statistical evidence of expression aftereffects for different identity trials, when there was a change in both surface texture and shape $[F(1,15)<1]$. The identity $\times$ expression interaction was not further modified by changes in gender between the adaptation and the test faces $[F(1,28)<1]$. This lack of an effect of gender in addition to that of identity may, however, reflect a floor effect, since there was little evidence of adaptation after identity change. Follow-up analyses revealed reliable same-identity (i.e., same-texture) adaptation even when the shape of the adapting and the test stimuli differed along the horizontal dimension $[F(1,15)=14.37$, $p<.002]$, with the magnitude of adaptation to a distorted surprise image $(M=8.64, S D=14.09)$ being highly similar to the magnitude of adaptation to the identical or undistorted anger image $(M=8.52, S D=10.61)$. This replicated the equal magnitude of adaptation for surprise and anger found in Experiment 1.

\section{Discussion}

Consistent with the first experiment, in Experiment 2, facial expression perception was noticeably biased by adaptation to images of facial expressions. That is, adapting to an expression pushed the category boundary toward the matching prototype, requiring the expression to be more prototypical to be correctly categorized. As in the first experiment, facial expression aftereffects were larger when the identities of the adapting and the test faces were the same. This identity modulation of expression adaptation was not an artifact of using the identical image in the same-identity condition, since a change in the horizontal aspect ratio between the surprise adaptation and the test faces in Experiment 2 resulted in robust adaptation of similar magnitude to adaptation found with identical anger adaptation and test images. In addition, identity influences on expression aftereffects remained when a computational model of expression appearance was used to hold expression geometry constant across identities. As such, differences in expression geometry in different individuals cannot fully account for the reduction in identity and expression cross-adaptation. Since identity information was retained despite identical expression structures, the decreased cross-adaptation likely reflects differences in the visual features supporting identity.

Of note was that the expression aftereffect, although still significant, was less statistically reliable in the second experiment than in the first. The difference was most likely due to changes in experimental design. First, the facial expression comparison was within subjects for Ex-

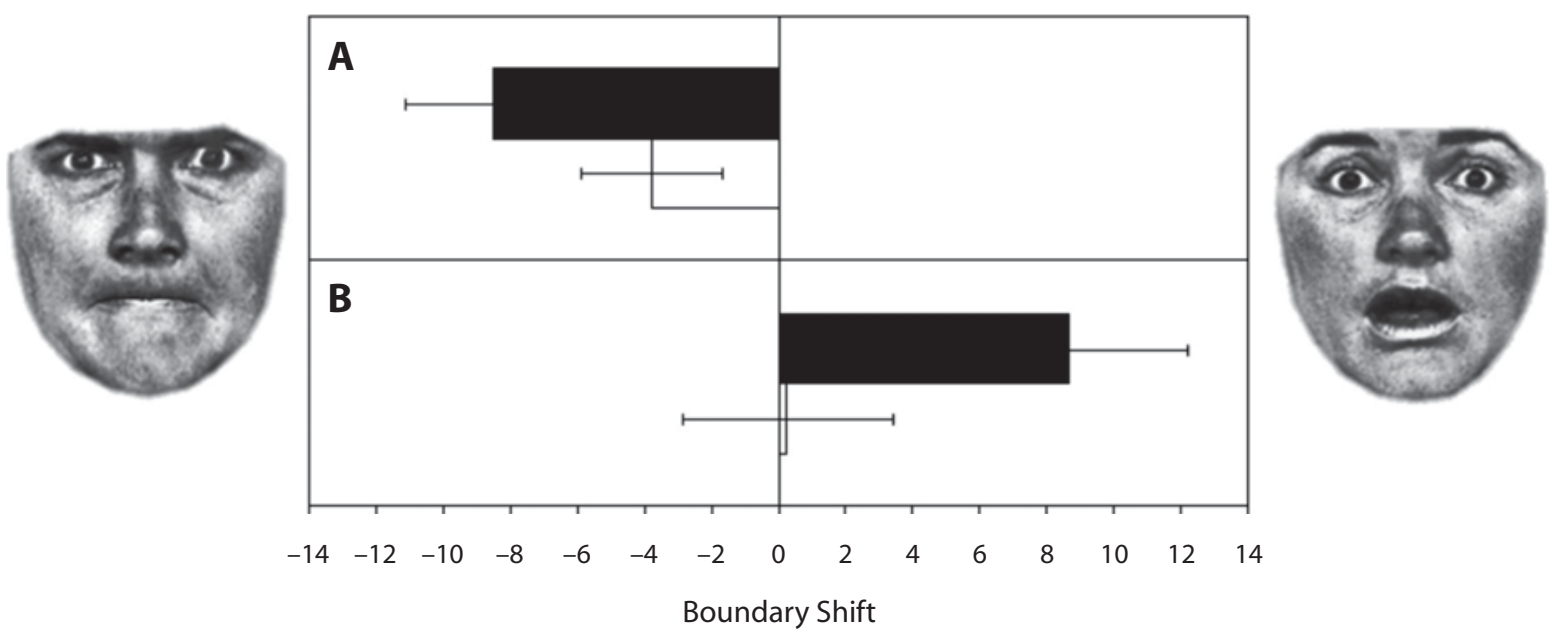

Figure 4. Mean difference between the pre- and the postadaptation category boundaries from Experiment 2 for the angersurprise continuum as a function of adapting expression $(\mathrm{A}=$ anger, $\mathrm{B}=$ surprise) and identity (black bars, same as the test face; white bars, different from the test face). A negative value represents a boundary shift toward the anger (left) end of the continuum, whereas a positive value represents a shift toward the surprise (right) end. Bar length represents magnitude of adaptation. Error bars represent the standard errors of the means. 
periment 1 and between subjects for Experiment 2, opting for adapting identity to be a within-subjects manipulation. Second, Experiment 1 contained twice the number of trials assessing the adaptation effect, since two continua were used (anger-surprise and disgust-fear) and only one continuum in Experiment 2 (anger-surprise). Nevertheless, despite this reduced expression adaptation in Experiment 2 , there remained a highly reliable influence of identity on expression adaptation.

\section{GENERAL DISCUSSION}

Our findings show that expression adaptation is reduced when identity is manipulated. This robust reduction in adaptation was present even when the shape of expression features were held constant (i.e., same expression geometry) across individuals. Reduced adaptation across identities provides support for partially overlapping neural processing of identity and facial expression. If identity and expression processing depend on entirely segregated streams of processing, altered identity should be represented as changes in a neural population vector in an identity-dedicated system, having minimal influence on expression-dedicated neural systems. As such, changes in identity will not result in changes in expression adaptation. In contrast to this neural model of independent expression and identity processing, identity changes had robust influences on expression adaptation. Nevertheless, there was evidence that adaptation was not entirely abolished following identity change. This is consistent with the presence of some level of identity-independent representation of emotional expression processing. Such findings are consistent with Calder and Young's (2005) proposal that identity and expression may be initially coded by a single representational system, rather than through separate processing routes involved in the extraction and separation of different facial cues. However, although Calder and Young suggested an overlap between the visual representations of expression and identity, this does not rule out the possibility that expression and identity are processed by separate higher order mechanisms involved in the interpretation of emotion or semantic information relating to identity. Thus, another account of the interaction between identity and expression is a feedforward mechanism or a common information-processing site (or sites) preceding higher level identity and expression analyses (e.g., Martínez, 2003).

Experiment 2 was an attempt to preserve facial features informative for expression perception by equating the geometrical shape features of the adapting and the test faces. This experiment was designed to manipulate surface reflectance information representing distinct identities while minimizing expression differences. Our results show that expression adaptation is attenuated across identities even when the shape features of the adapting face are equated to those of the continuum target face, thus indicating that the identity information in the texture interferes with expression adaptation. However, surface reflectance properties in the shape-normalized image may also convey important information for expression processing. In other words, expression information may also be carried by surface texture, in addition to shape features. Countering that notion, Calder, Burton, et al. (2001) showed that shape information (e.g., upturned and open lips) can be more informative than surface reflectance and texture information (e.g., bright teeth) for discriminating facial expressions in a PCA model. Much of the perceptually meaningful information conveyed by faces is in the phase structure, rather than the frequency domain, indicating that shape features (edges and discontinuities in the face image) are more important than detailed surface texture (Bartlett, Movellan, \& Sejnowski, 2002). In addition, computational systems employing local edge detection, such as Gabor wavelets (Littlewort, Bartlett, Fasel, Susskind, \& Movellan, 2006), perform well at expression recognition across a large degree of lighting conditions, also indicating the high information value of edge features. Nevertheless, surface reflectance contributes to expression perception, as has been supported by findings that a PCA representation combining shape and surface reflectance features performs better than either shape or reflectance alone (Calder, Burton, et al., 2001).

Whereas identity adaptation has been shown to be robust to certain transformations of the face (Jiang, Blanz, \& O'Toole, 2006), the present study indicates that expression adaptation is less so. This divergence may suggest that identity and expression features depend on distinct types of representations, counter to our suggestion of overlapping visual features in the neural processing of identity and expression. However, the nature of the transformations employed in these studies was quite distinct, with differences between identity and expression adaptation reflecting the use of rigid versus nonrigid transformations between adaptation and test stimuli. Previous studies in which identity adaptation has been examined have shown that identity adaptation is robust to rigid transformations, such as changes in viewpoint (Jiang et al., 2006). Changes in facial expression adaptation related to identity, such as those in the present experiments, reflect complex surface transformations between adaptation and test stimuli caused by nonrigid changes to the surface of the face. Thus, expression adaptation may be reduced by the nonrigid movement of facial features supporting identity and by associated nonrigid changes in texture. This was particularly striking in Experiment 2, in which the shape features of adapting and test stimuli were generated on the basis of an identical expression prototype. Despite the underlying commonality in expression geometry, expression adaptation was significantly disrupted. That is, large reductions in expression adaptation were found even when the underlying shape of the features was held constant (i.e., shape of eyes, nose, mouth, outline of face) but surface texture features supporting identity were varied. This suggests that changes in texture and reflectance caused by nonrigid changes to the surface of the face may affect both identity and expression adaptation, as is predicted by PCA applied to face images (Calder, Burton, et al., 2001). However, it is important to consider whether rigid transformations (e.g., viewpoint) will also alter expression adaptation. Future studies will need to demonstrate that expression adaptation is robust to rigid transformations, 
further suggesting that expression and identity representations call upon shared neural substrates.

One concern is that facial adaptation may influence neither identity nor facial expression processing themselves but may influence an earlier common visual representation. That is, identity and expression processing may occur in parallel at an abstract, high level of processing at neural loci downstream of facial adaptation effects. By contrast, the neural locus of the intersection of identity and expression as revealed through facial adaptation is unlikely at very low levels of visual representation, since visual fixation was not maintained through the adaptation and test phases. Consistent with an intermediate- to high-level locus, as was mentioned above, identity adaptation has been shown to generalize across viewpoint (Jiang et al., 2006). This suggests that facial adaptation is at a level above view-dependent coding, where these lower order representations are integrated into a viewindependent representation of identity. We propose that it is this level that represents the common neural locus of adaptation between identity and expression. However, as was mentioned above, it will be of interest in future studies to establish that expression adaptation indeed occurs at a similar view-independent level of representation.

Where is the neural representation supporting expression adaptation? The separation between the processing of static invariant and dynamic variable aspects of faces has been proposed to involve the inferior temporal gyrus (the lateral fusiform gyrus in humans; Grill-Spector et al., 2004; Kanwisher et al., 1997) and the STS (Haxby et al., 2000; Narumoto et al., 2001; Winston et al., 2004). Neuroimaging evidence suggests that the high-level interactions between identity and facial expression likely occur in the latter. The STS contains cells sensitive to both expression and identity (Perrett et al., 1984) and also exhibits adaptation to both facial characteristics (Winston et al., 2004). Specifically, the posterior STS has shown adaptation to identity and (to a lesser degree) expression, whereas the middle STS has shown adaptation to expression but not to identity (Winston et al., 2004). Fox and Barton (2007b) proposed an identity-specific neural representation of expression corresponding to a neural population that adapts to both identity and expression (i.e., posterior STS) and an identity-independent expression representation corresponding to a neural population that adapts to expression but not to identity (i.e., the middle STS). The present results suggest that adaptation to facial expressions depends conjointly on the posterior and the middle STS, with the former representing identity-based expression adaptation and the latter representing more abstract expression-based adaptation. The reduction in adaptation we observed related to changing identity is consistent with evidence that expression adaptation depends to a large degree on the posterior STS. However, since this reduction was not always absolute, the amount of spared adaptation across individuals may depend on the middle STS. That the STS is responsive to both identity and expression, in combination with the results of research showing that cells in the STS are also responsive to biological motion (Grossman et al., 2000; Servos, Osu, Santi, \& Kawato, 2002), is also consistent with the alternative proposal of a common motion estimation process (with the STS as a possible neural substrate) mediating the interaction between identity and expression processes (Martínez, 2003).

The possibility of identity-dependent and -independent representations suggests that there is some form of functional asymmetry in how identity and facial expression coding overlap, wherein identity processing is relatively view (e.g., Jiang et al., 2006) and expression (e.g., Fox \& Barton, 2007a) independent but expression processing may be relatively view and identity dependent. Indeed, identity and familiarity have been shown to modulate judgments of facial expression, whereas judgments of identity are unaffected by expression, suggesting an asymmetric relationship between identity and expression (Schweinberger \& Soukop, 1998). It may be that expression processing relies more on integrative mechanisms than does identity processing (Calder \& Young, 2005). As compared with identity recognition, for which there is a scarcity of evidence that facial identity interacts with other facial cues, expression recognition may make use of information from a variety of sources. This is suggested by evidence for multiple systems for the processing of expressions and emotions (e.g., Adolphs et al., 1999; Phillips et al., 1998), for the multimodal analysis of expression (i.e., convergence of facial, vocal, and dynamic components; e.g., de Gelder \& Vroomen, 2000; Dolan, Morris, \& de Gelder, 2001), and for the co-processing of expression with other facial cues from dynamic sources, such as gaze and lip speech (e.g., Adams \& Kleck, 2003, 2005), much of which is supported by the STS (Haxby et al., 2000). Whereas dynamic cues need to be constantly monitored during social interaction, this is not true for static facial identity. The dependence on such dynamic integrative mechanisms for expression, relative to identity processing, suggests a functional and, thus, neuroanatomic asymmetry. Whereas expression processing may be sensitive to changes of many sorts, including identity - a situation rarely encountered by perceptual systems - identity processing may be relatively robust to dynamic changes in the face, such as expression - a situation encountered in all social interactions.

\section{AUTHOR NOTE}

This work was supported by grants from the Natural Sciences and Engineering Research Council and Canada Research Chairs program to A.K.A. Please address correspondence to A. K. Anderson, Department of Psychology, University of Toronto, 100 St. George Street, Toronto, ON, M5A 3J8 Canada (e-mail: anderson@psych.utoronto.ca).

\section{REFERENCES}

Adams, R. B., \& KLeck, R. E. (2003). Perceived gaze direction and the processing of facial displays of emotion. Psychological Science, 14, 644-647.

AdAms, R. B., \& KLECK, R. E. (2005). The effects of direct and averted gaze on the perception of facially communicated emotion. Emotion, 5, 3-11.

Adolphs, R., Tranel, D., Hamann, S., Young, A. W., Calder, A. J., Phelps, E. A., ET AL. (1999). Recognition of facial emotion in nine individuals with bilateral amygdala damage. Neuropsychologia, 37, 1111-1117. 
Anderson, A. K., Christoff, K., Panitz, D., De Rosa, E., \& GabriELI, J. D. E. (2003). Neural correlates of the automatic processing of threat facial signals. Journal of Neuroscience, 23, 5627-5633.

Bartlett, M. S., Movellan, J. R., \& Sejnowski, T. J. (2002). Face recognition by independent component analysis. IEEE Transactions on Neural Networks, 13, 1450-1464.

Baudouin, J.-Y., Gilibert, D., Sansone, S., \& Tiberghien, G. (2000). When the smile is a cue to familiarity. Memory, 8, 285-292.

BRUCE, V. (1986). Influences of familiarity on the processing of faces. Perception, 15, 387-397.

Bruce, V., \& Young, A. (1986). Understanding face recognition. British Journal of Psychology, 77, 305-327.

Bruyer, R., Laterre, C., Seron, X., Feyereisen, P., Strypstein, E., Pierrard, E., \& Rectem, D. (1983). A case of prosopagnosia with some preserved covert remembrance of familiar faces. Brain \& $\mathrm{Cog}$ nition, 2, 257-284.

Calder, A. J., Beaver, J. D., Winston, J. S., Dolan, R. J., Jenkins, R., Eger, E., \& Henson, R. N. A. (2007). Separate coding of different gaze directions in the superior temporal sulcus and inferior parietal lobule. Current Biology, 17, 20-25.

Calder, A. J., Burton, A. M., Miller, P., Young, A. W., \& AKaMATSU, S. (2001). A principal component analysis of facial expressions. Vision Research, 41, 1179-1208.

Calder, A. J., Lawrence, A. D., \& Young, A. W. (2001). Neuropsychology of fear and loathing. Nature Reviews Neuroscience, 2, 352-363.

Calder, A. J., \& Young, A. W. (2005). Understanding the recognition of facial identity and facial expression. Nature Reviews Neuroscience, 6, 641-651.

Cornsweet, T. N. (1962). The staircase-method in psychophysics. American Journal of Psychology, 75, 485-491.

Craw, I., \& Cameron, P. (1991). Parameterising images for recognition and reconstruction. In P. Mowforth (Ed.), Proceedings of the 2nd British Machine Vision Conference (pp. 367-370). London: Springer.

de Gelder, B., \& Vroomen, J. (2000). The perception of emotions by ear and by eye. Cognition \& Emotion, 14, 289-311.

Dolan, R. J., Morris, J. S., \& de Gelder, B. (2001). Crossmodal binding of fear in voice and face. Proceedings of the National Academy of Sciences, 98, 10006-10010.

Ekman, P., \& Friesen, W. V. (1976). Pictures of facial affect. Palo Alto, CA: Consulting Psychologists Press.

Fox, C. J., \& BARTON, J. J. S. (2007a). Asymmetric relationship in representations of facial identity and expression for novel faces within the human visual system [Abstract]. Journal of Vision, 7(9), 997a.

Fox, C. J., \& Barton, J. J. S. (2007b). What is adapted in face adaptation? The neural representations of expression in the human visual system. Brain Research, 1127, 80-89.

Ganel, T., \& Goshen-GotTstein, Y. (2004). Effects of familiarity on the perceptual integrality of the identity and expression of faces: The parallel-route hypothesis revisited. Journal of Experimental Psychology: Human Perception \& Performance, 30, 583-597.

Goodall, C. (1991). Procrustes methods in the statistical analysis of shape. Journal of the Royal Statistical Society: Series B, 53, 285-339.

Goshtasby, A. (1986). Piecewise linear mapping functions for image registration. Pattern Recognition, 19, 459-466.

Grill-Spector, K., Henson, R., \& Martin, A. (2006). Repetition and the brain: Neural models of stimulus-specific effects. Trends in Cognitive Sciences, 10, 14-23.

Grill-Spector, K., Knouf, N., \& Kanwisher, N. (2004). The fusiform face area subserves face perception, not generic within-category identification. Nature Reviews Neuroscience, 7, 555-562.

Grossman, E., Donnelly, M., Price, R., Pickens, P., Morgan, V., Neighbor, G., \& Blake, R. (2000). Brain areas involved in perception of biological motion. Journal of Cognitive Neuroscience, 12, 711-720.

Haxby, J. V., Hoffman, E. A., \& Gobbini, M. I. (2000). The distributed human neural system for face perception. Trends in Cognitive Sciences, 4, 223-233.

Hsu, S.-M., \& Young, A. W. (2004). Adaptation effects in facial expression recognition. Visual Cognition, 11, 871-899.
Jenkins, R., BeAver, J. D., \& CALDER, A. J. (2006). I thought you were looking at me: Direction-specific aftereffects in gaze perception. Psychological Science, 17, 506-513.

Jiang, F., Blanz, V., \& O'Toole, A. J. (2006). Probing the visual representation of faces with adaptation: A view from the other side of the mean. Psychological Science, 17, 493-500.

Kanwisher, N., McDermott, J., \& Chun, M. M. (1997). The fusiform face area: A module in human extrastriate cortex specialized for face perception. Journal of Neuroscience, 17, 4302-4311.

Kaufmann, J. M., \& Schweinberger, S. R. (2004). Expression influences the recognition of familiar faces. Perception, 33, 399-408.

KöHLER, W., \& Wallach, H. (1944). Figural aftereffects: An investigation of visual processes. Proceedings of the American Philosophical Society, 88, 269-357.

Kurucz, J., \& Feldmar, G. (1979). Prosopo-affective agnosia as a symptom of cerebral organic disease. Journal of the American Geriatrics Society, 27, 225-230.

Kurucz, J., Feldmar, G., \& Werner, W. (1979). Prosopo-affective agnosia associated with chronic organic brain syndrome. Journal of the American Geriatrics Society, 27, 91-95.

LaBar, K. S., Crupain, M. J., Voyvodic, J. T., \& McCarthy, G. (2003). Dynamic perception of facial affect and identity in the human brain. Cerebral Cortex, 13, 1023-1033.

Leopold, D. A., O'Toole, A. J., Vetter, T., \& Blanz, V. (2001). Prototype-referenced shape encoding revealed by high-level aftereffects. Nature Neuroscience, 4, 89-94.

Littlewort, G., Bartlett, M. S., Fasel, I., Susskind, J., \& MovelLAN, J. (2006). Dynamics of facial expression extracted automatically from video. Image \& Vision Computing, 24, 615-625.

Martínez, A. M. (2003). Matching expression variant faces. Vision Research, 43, 1047-1060.

Narumoto, J., Okada, T., Sadato, N., Fukui, K., \& Yonekura, Y. (2001). Attention to emotion modulates fMRI activity in human right superior temporal sulcus. Cognitive Brain Research, 12, 225-231.

Perrett, D. I., Smith, P. A. J., Potter, D. D., Mistlin, A. J., Head, A. S., Milner, A. D., \& JeEves, M. A. (1984). Neurons responsive to faces in the temporal cortex: Studies of functional organization, sensitivity to identity, and relation to perception. Human Neurobiology, 3, 197-208.

Phillips, M. L., Young, A. W., Scott, S. K., Calder, A. J., Andrew, C., Giampietro, V., ET AL. (1998). Neural responses to facial and vocal expressions of fear and disgust. Proceedings of the Royal Society B, 265, 1809-1817.

Rhodes, G., JefFery, L., Watson, T. L., Clifford, C. W. G., \& NAKAYAMA, K. (2003). Fitting the mind to the world: Face adaptation and attractiveness aftereffects. Psychological Science, 14, 558-566.

Schweinberger, S. R., \& Soukup, G. R. (1998). Asymmetric relationships among perceptions of facial identity, emotion, and facial speech. Journal of Experimental Psychology: Human Perception \& Performance, 24, 1748-1765.

Servos, P., Osu, R., Santi, A., \& Kawato, M. (2002). The neural substrates of biological motion perception: An fMRI study. Cerebral Cortex, 12, 772-782.

Susskind, J. M., Littlewort, G., Bartlett, M. S., Movellan, J., \& ANDERSON, A. K. (2007). Human and computer recognition of facial expressions of emotion. Neuropsychologia, 45, 152-162.

Walker, S., Bruce, V., \& O’Malley, C. (1995). Facial identity and facial speech processing: Familiar faces and voices in the McGurk effect. Perception \& Psychophysics, 57, 1124-1133.

Webster, M. A., Kaping, D., Mizokami, Y., \& Duhamel, P. (2004). Adaptation to natural facial categories. Nature, 428, 557-561.

Webster, M. A., \& MacLin, O. H. (1999). Figural aftereffects in the perception of faces. Psychonomic Bulletin \& Review, 6, 647-653.

Winston, J. S., Henson, R. N. A., Fine-Goulden, M. R., \& Dolan, R. J. (2004). fMRI-adaptation reveals dissociable neural representations of identity and expression in face perception. Journal of Neurophysiology, 92, 1830-1839.

Zhao, L., \& Chubb, C. (2001). The size-tuning of the face-distortion aftereffect. Vision Research, 41, 2979-2994.

(Manuscript received December 14, 2007; revision accepted for publication February 23, 2008.) 